\title{
Elementary numerosity and measures
}

\author{
VIERI BENCI \\ EMANUELE BOTTAZZI \\ MAURO DI NASSO
}

\begin{abstract}
In this paper we introduce the notion of elementary numerosity as a special function defined on all subsets of a given set $\Omega$ which takes values in a suitable non-Archimedean field and satisfies the same formal properties as finite cardinality. By improving a classic result by $\mathrm{C}$. W. Henson in nonstandard analysis, we prove a general compatibility result between such elementary numerosities and measures.
\end{abstract}

2010 Mathematics Subject Classification 26E30; 28E15; 26E35; 28E15; 60A05 (primary); (secondary)

Keywords: Non-Archimedean mathematics, measure theory, nonstandard analysis, numerosities.

\section{Introduction}

In mathematics there are essentially two main ways to estimate the size of a set, depending on whether one is working in a discrete or in a continuous setting. In the continuous case one uses the notion of (finitely) additive measure, namely a real-valued function (possibly taking also the value $+\infty$ ) which satisfies the following properties:

(1) $m(\emptyset)=0$

(2) $m(A) \geq 0$

(3) $m(A \cup B)=m(A)+m(B)$ whenever $A \cap B=\emptyset$.

In the discrete case one uses the notion of cardinality $\mathfrak{n}$ that strengthens the three properties itemized above as follows:

(n.1) $\mathfrak{n}(\emptyset)=0$

(n.2) $\mathfrak{n}(A) \geq 0$

(n.3) $\mathfrak{n}(A \cup B)=\mathfrak{n}(A)+\mathfrak{n}(B)$ whenever $A \cap B=\emptyset$ 
(n.4) $\mathfrak{n}(\{x\})=1$ for all singletons.

The goal of this paper is to investigate the relationships between these two notions. To this end, we will introduce the concept of elementary numerosity as a special function defined on all subsets of a given set $\Omega$ that takes values in a suitable ordered field $\mathbb{F}$ and satisfies the four properties of finite cardinalities itemized above (see Definition 1.1). We remark that if $\Omega$ is infinite, then the range of such a function $\mathfrak{n}$ necessarily contains infinite numbers, and hence the field $\mathbb{F}$ must be non-Archimedean. Notice that Cantorian cardinality also satisfies properties $(\mathfrak{n} .1),(\mathfrak{n} .2),(\mathfrak{n} .3),(\mathfrak{n} .4)$, the fundamental difference being that "numerosities" are required to be elements of an ordered field.

We will assume the reader to be familiar with the basics of nonstandard analysis; a classic reference is Davis [6] (see also the more recent book by Goldblatt [8]). For the used terminology of measure theory, we refer to Yeh [11]. A comprehensive exposition of nonstandard measure theory and probability theory is given in Ross [14].

Acknowledgement. The authors thank the editor D.A. Ross and the anonymous referees for several useful remarks on previous versions of this paper, and for pointing out relevant bibliographic references.

\section{Elementary numerosity}

The notion of numerosity was introduced in Benci [1] and in Benci and Di Nasso [3] as a generalization of finite cardinality that also applies to infinite sets. (Its theory has then been developed in a series of papers; see eg [4] and [7]). The main feature of numerosities is that they preserve the spirit of the ancient Euclidean principle that "the whole is larger than the part"; indeed, the numerosity of a proper subset is strictly smaller than the numerosity of the whole set. Inspired by the same idea, we now aim at refining the notion of finitely additive measure in such a way that also single points count. To this end, we will consider ordered fields $\mathbb{F} \supset \mathbb{R}$ which properly extend the real line. We remark that such fields are necessarily non-Archimedean, ie they contain infinitesimal numbers $\epsilon \neq 0$ such that $-1 / n<\epsilon<1 / n$ for all $n \in \mathbb{N}$. Following the same terminology used in nonstandard analysis, we say that two elements $\xi, \zeta \in \mathbb{F}$ are infinitely close, and write $\xi \approx \zeta$, when their difference $\xi-\zeta$ is infinitesimal; we say that a number $\xi \in \mathbb{F}$ is finite when $-n<\xi<n$ for some $n \in \mathbb{N}$, and is infinite otherwise. By the completeness property of $\mathbb{R}$ it is easily verified that every finite $\xi \in \mathbb{F}$ is infinitely close to a unique real number $r$, namely $r=\inf \{x \in \mathbb{R} \mid x>\xi\}$. Such a number $r$ is called the standard part of $\xi$, and we use notation $r=\operatorname{st}(\xi)$. The 
standard part is extended to all numbers in $\mathbb{F}$ by setting $\operatorname{st}(\xi)=+\infty$ when $\xi$ is infinite and positive, and $\operatorname{st}(\xi)=-\infty$ when $\xi$ is infinite and negative.

Definition 1.1 An elementary numerosity on a set $\Omega$ is a function

$$
\mathfrak{n}: \mathcal{P}(\Omega) \rightarrow[0,+\infty)_{\mathbb{F}}
$$

defined on all subsets of $\Omega$, taking values in the non-negative part of an ordered field $\mathbb{F} \supseteq \mathbb{R}$, and such that the following two conditions are satisfied:

(1) $\mathfrak{n}(\{x\})=1$ for every point $x \in \Omega$;

(2) $\mathfrak{n}(A \cup B)=\mathfrak{n}(A)+\mathfrak{n}(B)$ whenever $A$ and $B$ are disjoint.

Elementary numerosities satisfy the same basic properties as finite cardinalities. Indeed:

Proposition 1.2 Let $\mathfrak{n}$ be an elementary numerosity. Then:

(1) $\mathfrak{n}(A)=0$ if and only if $A=\emptyset$;

(2) If $A \subset B$ is a proper subset, then $\mathfrak{n}(A)<\mathfrak{n}(B)$;

(3) If $F$ is a finite set of cardinality $n$, then $\mathfrak{n}(F)=n$.

Proof Notice that $\mathfrak{n}(\emptyset)=\mathfrak{n}(\emptyset \cup \emptyset)=\mathfrak{n}(\emptyset)+\mathfrak{n}(\emptyset)$, and $x=0$ is the only number $x \in \mathbb{F}$ such that $x+x=x$. If $A \subseteq B$ then $\mathfrak{n}(B)=\mathfrak{n}(A)+\mathfrak{n}(B \backslash A) \geq \mathfrak{n}(A)$. Moreover, if $A \subset B$ is a proper subset and $x \in B \backslash A$, then $\mathfrak{n}(B) \geq \mathfrak{n}(A \cup\{x\})=\mathfrak{n}(A)+\mathfrak{n}(\{x\})=$ $\mathfrak{n}(A)+1>\mathfrak{n}(A)$. In consequence, $\mathfrak{n}(A)>0$ for all nonempty sets $A$. Finally, the last property directly follows by additivity and the fact that every singleton has numerosity 1 .

Remark 1.3 If one takes $\mathbb{F}=\mathbb{R}$, then elementary numerosities $\mathfrak{n}$ exist on a set $\Omega$ if and only if $\Omega$ is finite; and in this case, the only numerosity $\mathfrak{n}$ is given by the finite cardinality.

We will show in the sequel that by taking suitable non-Archimedean fields that properly extend the real line, elementary numerosities exist on every infinite set.

Given an elementary numerosity and a "measure unit" $\beta>0$, there is a canonical way to construct a (real-valued) finitely additive measure. 
Definition 1.4 Let $\mathfrak{n}: \mathcal{P}(\Omega) \rightarrow[0,+\infty)_{\mathbb{F}}$ be an elementary numerosity, and let $\beta \in \mathbb{F}$ be a positive number. The function $\mu_{\mathfrak{n}, \beta}: \mathcal{P}(\Omega) \rightarrow[0,+\infty]_{\mathbb{R}}$ is defined by setting

$$
\mu_{\mathfrak{n}, \beta}(A)=\text { st }\left(\frac{\mathfrak{n}(A)}{\beta}\right) \text {. }
$$

It is easily verified from the properties of the standard map st that $\mu_{\mathfrak{n}, \beta}$ is a finitely additive measure. Moreover, notice that $\mu_{\mathfrak{n}, \beta}$ is non-atomic if and only if $\mu_{\mathfrak{n}, \beta}(\{x\})=$ $\operatorname{st}(1 / \beta)=0$ for all singletons, and this holds if and only if $\beta$ is infinite.

The class of measures that we just introduced turns out to be really general. Indeed, the goal of this paper is to prove a strong version of the following

- Claim. Every finitely additive non-atomic measure is a restriction of a suitable $\mu_{\mathfrak{n}, \beta}$

\section{The main result}

In the 60s and early 70s of the last century, researchers in nonstandard analysis deeply investigated the possibility of representing finitely additive measures as counting measures on suitable hyperfinite samples. ${ }^{1}$ The starting point was the following key observation, pointed out by A.R. Bernstein and F. Wattenberg in [5]:

- For every nonempty hyperfinite set $F \subseteq{ }^{*} \Omega$,

$$
\mu_{F}(A)=s t\left(\frac{\left\|^{*} A \cap F\right\|}{\|F\|}\right)
$$

is a finitely additive probability measure defined on all subsets of $\Omega{ }^{2}$

In that paper, the tool of hyperfinite counting measures was used to give a nonstandard proof of the existence of a totally defined translation-invariant extension of the Lebesgue measure on $\mathbb{R}$. In 1972, extending a previous result obtained by A. Robinson [13], C.W. Henson proved the following general representation theorem:

- (Theorem 1 of [10]) Let $\Omega$ be an infinite set and assume that the nonstandard extension $*$ satisfies the property of $\left(2^{|\Omega|}\right)^{+}$-enlargement. If $m$ is a non-atomic finitely additive probability measure defined on all subsets of $\Omega$, then there exists a nonempty hyperfinite set $F \subseteq{ }^{*} \Omega$ such that $m=\mu_{F}$.

\footnotetext{
${ }^{1}$ After the introduction of the Loeb measure [12] in 1975, this line of research has been almost abandoned; however, see D.A. Ross' paper [15] for a survey of alternative nonstandard approaches to measure theory.

${ }^{2}$ By $\|\cdot\|$ is denoted the hyperfinite internal cardinality.
} 
Remark 2.1 In any model of nonstandard analysis, every hyperfinite set $F \subseteq{ }^{*} \Omega$ such that ${ }^{*} x \in F$ for all $x \in \Omega$ determines an elementary numerosity $\mathfrak{n}_{F}: \mathcal{P}(\Omega) \rightarrow{ }^{*} \mathbb{R}$ simply by letting:

$$
\mathfrak{n}_{F}(A)=\left\|^{*} A \cap F\right\|
$$

In consequence, by taking ratios of the elementary numerosity $\mathfrak{n}=\mathfrak{n}_{F}$ to the fixed "measure unit" $\beta=\|F\|>0$, the above Henson's Theorem yields the following corollary:

- For every non-atomic finitely additive probability measure $(\Omega, \mathcal{P}(\Omega), \mu)$ defined on all subsets of a set $\Omega$, there exist an ordered field of hyperreals $* \mathbb{R}$, an elementary numerosity $\mathfrak{n}: \mathcal{P}(\Omega) \rightarrow[0,+\infty)_{*} \mathbb{R}$, and a positive number $\beta \in{ }^{*} \mathbb{R}$, such that $\mu=\mu_{\mathfrak{n}, \beta}$.

The above result shows that elementary numerosities can be found which are compatible with any given non-atomic finitely additive probability measure provided one takes ratios over a suitable measure unit and identities are taken only up to infinitesimals. Here, we investigate the possibility of a stronger coherence of elementary numerosities with measures. Most notably, a natural requirement would be to have equal numerosity for sets of equal measure.

- Given a non-atomic finitely additive measure $\mu$ defined on an algebra $\mathfrak{A} \subseteq$ $\mathcal{P}(\Omega)$, is there an elementary numerosity $\mathfrak{n}: \mathcal{P}(\Omega) \rightarrow[0,+\infty)_{\mathbb{F}}$ which is "coherent" with $\mu$, in the following strong sense?

(1) There exists a positive $\beta$ such that $\mu_{\mathfrak{n}, \beta}(A)=\mu(A)$ for all $A \in \mathfrak{A}$;

(2) $\mu(A)=\mu\left(A^{\prime}\right) \Longleftrightarrow \mathfrak{n}(A)=\mathfrak{n}\left(A^{\prime}\right)$ for all $A, A^{\prime} \in \mathfrak{A}$.

Unfortunately, in the presence of nonempty null sets or of sets of infinite measure, it is readily seen that (2) cannot hold in general. (Recall that proper subsets have a strictly smaller numerosity.) However, these are basically the only obstacles; indeed, for any given measure, we will be able to find elementary numerosities that satisfy (1) and that satisfy also the "strong" coherence property (2) on suitable subalgebras. To this end, we will prove an improvement of Henson's Theorem about nonstandard representation of measures, as given by the theorem below.

We remark that our proof is grounded on a combinatorial lemma, and uses different arguments with respect to the ones used in the original proofs of the classic results by Bernstein, Wattenberg and Henson.

Since the proof is rather long, it is put off to Section 3. 
Theorem 2.2 Let $(\Omega, \mathfrak{A}, \mu)$ be a non-atomic finitely additive measure on the infinite set $\Omega$, and let $\mathfrak{B} \subseteq \mathfrak{A}$ be a subalgebra that does not contain nonempty null sets. Then in any model of nonstandard analysis that satisfies the property of $\left(2^{|\Omega|}\right)^{+}$-enlargement there exists a hyperfinite set $F \subseteq{ }^{*} \Omega$ such that:

(1) ${ }^{*} x \in F$ for every $x \in \Omega$;

(2) $\left\|F \cap{ }^{*} B\right\|=\left\|F \cap{ }^{*} B^{\prime}\right\| \Leftrightarrow \mu(B)=\mu\left(B^{\prime}\right)$ for all $B, B^{\prime} \in \mathfrak{B}$ of finite measure;

(3) for every hyperreal number of the form $\beta=\frac{\left\|F \cap^{*} Z\right\|}{\mu(Z)}$ where $Z \in \mathfrak{A}$ has positive finite measure, and for every $A \in \mathfrak{A}$ :

$$
\mu(A)=s t\left(\frac{\|F \cap * A\|}{\beta}\right) .
$$

Let us see now the relevant corollary about elementary numerosities.

Theorem 2.3 Let $(\Omega, \mathfrak{A}, \mu)$ and $\mathfrak{B} \subseteq \mathfrak{A}$ satisfy the hypotheses of Theorem 2.2. Then there exists an elementary numerosity $\mathfrak{n}$ on $\Omega$ such that:

(1) $\mu(B)=\mu\left(B^{\prime}\right) \Leftrightarrow \mathfrak{n}(B)=\mathfrak{n}\left(B^{\prime}\right)$ for all $B, B^{\prime} \in \mathfrak{B}$ of finite measure;

(2) For every $\beta=\mathfrak{n}(Z) / \mu(Z)$ where $Z \in \mathfrak{A}$ has positive finite measure, $\mu=\left(\mu_{\mathfrak{n}, \beta}\right)_{\mid \mathfrak{A}}$ is the restriction of $\mu_{\mathfrak{n}, \beta}$ to $\mathfrak{A}$.

Proof Let $F \subseteq{ }^{*} \Omega$ be the hyperfinite set as given by Theorem 2.2, and let $\mathfrak{n}=\mathfrak{n}_{F}$.

We stress the fact that in (2), the measure $\mu_{\mathfrak{n}, \beta}$ that represents $\mu$ does not depend on the choice of $\beta$, as long as $\beta=\mathfrak{n}(Z) / \mu(Z)$ for some $Z$ with $0<\mu(Z)<+\infty$.

In several examples, one naturally finds subalgebras $\mathfrak{B}$ with the property that every nonempty $B \in \mathfrak{B}$ has positive measure. For instance, if one considers the Lebesgue measure $m$ on $\mathbb{R}$ then one can take $\mathfrak{B}$ as the algebra of the finite unions of half-open intervals $[a, b)$ where possibly $b=+\infty$, and intervals of the form $(-\infty, b)$ where possibly $b=+\infty$. In this case, the above theorem guarantees the existence of an elementary numerosity $\mathfrak{n}$ defined on all subsets of $\mathbb{R}$ such that $m(A) \approx \mathfrak{n}(A) / \mathfrak{n}([0,1))$ for all Lebesgue measurable $A \subseteq \mathbb{R}$, and with the strong translation-invariant property that $\mathfrak{n}([a, a+\ell))=\mathfrak{n}([b, b+\ell))$ for every $a, b$ and for every length $\ell>0$. This example, along with others, is studied in Benci, Bottazzi and Di Nasso [2].

Remark 2.4 Notice that Theorem 2.3 still does not provide a full proof for our claim (made at the end of Section 1) that every finitely additive non-atomic measure $(\Omega, \mathfrak{A}, \mu)$ be a restriction of a measure of the form $\mu_{\mathfrak{n}, \beta}$. Indeed, if $\mu$ only takes the values 0 
and $+\infty$, then there are no suitable "measure units" $\beta$, because there are no sets $Z \in \mathfrak{A}$ with positive finite measure. Nevertheless, we remark that even such measures are restrictions of suitable $\mu_{\mathfrak{n}, \beta}$. To see this, pick any non-atomic finitely additive probability measure $\left(\Omega^{\prime}, \mathcal{P}\left(\Omega^{\prime}\right), \mu^{\prime}\right)$ where $\Omega^{\prime} \cap \Omega=\emptyset$. Then let

$$
\mathfrak{C}=\left\{A \cup B \mid A \in \mathfrak{A}, B \in \mathcal{P}\left(\Omega^{\prime}\right)\right\},
$$

and define $\nu: \mathfrak{C} \rightarrow[0,+\infty]_{\mathbb{R}}$ by putting

$$
\nu(C)=\mu(C \cap \Omega)+\mu^{\prime}\left(C \cap \Omega^{\prime}\right) .
$$

It is easily verified that $\nu$ is a non-atomic finitely additive measure over $\Omega \cup \Omega^{\prime}$; notice also that $\nu\left(\Omega^{\prime}\right)=\mu^{\prime}\left(\Omega^{\prime}\right)=1$. So, Theorem 2.3 can be applied to $\nu$ and we obtain the existence of an elementary numerosity $\mathfrak{n}$ and of a number $\beta$ (eg, $\left.\beta=\mathfrak{n}\left(\Omega^{\prime}\right) / \nu\left(\Omega^{\prime}\right)=\mathfrak{n}\left(\Omega^{\prime}\right)\right)$ such that $\mu_{\mathfrak{n}, \beta}(C)=\nu(C)$ for all $C \in \mathfrak{C}$. In particular, $\mu_{\mathfrak{n}, \beta}(A)=\mu(A)$ for all $A \in \mathfrak{A}$, as desired.

\section{Proof of the Main Theorem 2.2}

The proof of the main theorem is grounded on the following combinatorial property.

Lemma 3.1 Let $(\Omega, \mathfrak{A}, \mu)$ be a non-atomic finitely additive measure, and let $\mathfrak{B} \subseteq \mathfrak{A}$ be a subalgebra of subsets of $\Omega$ whose nonempty sets have all positive measure. Denote by $\mathfrak{A}_{f}$ (by $\mathfrak{B}_{f}$ ) the family of sets in $\mathfrak{A}$ (in $\mathfrak{B}$, respectively) which have finite measure. Given $m \in \mathbb{N}$, finitely many points $x_{1}, \ldots, x_{k} \in \Omega$, and finitely many nonempty sets $A_{1}, \ldots, A_{v} \in \mathfrak{A}$, there exists a finite subset $\lambda \subset \Omega$ that satisfies the following properties:

(1) $x_{1}, \ldots, x_{k} \in \lambda$;

(2) If $A_{i}, A_{j} \in \mathfrak{B}_{f}$ are nonempty sets such that $\mu\left(A_{i}\right)=\mu\left(A_{j}\right)$, then $\left|\lambda \cap A_{i}\right|=$ $\left|\lambda \cap A_{j}\right|$

(3) If $A_{i} \in \mathfrak{A}_{f}$ and $\mu\left(A_{i}\right) \neq 0$, then for all $j$ such that $A_{j} \in \mathfrak{A}_{f}$ :

$$
\left|\frac{\left|\lambda \cap A_{j}\right|}{\left|\lambda \cap A_{i}\right|}-\frac{\mu\left(A_{j}\right)}{\mu\left(A_{i}\right)}\right|<\frac{1}{m}
$$

(4) If $A_{i} \in \mathfrak{A}_{f}$ and $\mu\left(A_{i}\right) \neq 0$, then for all $j$ such that $A_{j} \in \mathfrak{A} \backslash \mathfrak{A}_{f}$ :

$$
\frac{\left|\lambda \cap A_{j}\right|}{\left|\lambda \cap A_{i}\right|}>m
$$


Proof Without loss of generality, we can assume that the given sets $A_{i}$ are arranged in such a way that $A_{1}, \ldots, A_{l} \in \mathfrak{B}_{f}, A_{l+1}, \ldots, A_{n} \in \mathfrak{A}_{f} \backslash \mathfrak{B}_{f}$ and $A_{n+1}, \ldots, A_{v} \in \mathfrak{A} \backslash \mathfrak{A}_{f}$ for suitable $l$ and $n$. It will be convenient in the sequel that the considered elements in $\mathfrak{B}_{f}$ be pairwise disjoint. To this end, consider the partition $\left\{B_{1}, \ldots, B_{h}\right\}$ induced by $\left\{A_{1}, \ldots, A_{l}\right\} .{ }^{3}$ Notice that, by the algebra properties of $\mathfrak{B}$, every piece $B_{s}$ belongs to $\mathfrak{B}_{f}$. Finally, let

$$
\bigcup_{i=1}^{n} A_{i}=C_{1} \sqcup \ldots \sqcup C_{p} \sqcup D_{1} \sqcup \ldots \sqcup D_{q}
$$

be the partition of $\bigcup_{i=1}^{n} A_{i}$ induced by $\left\{B_{1}, \ldots, B_{h}, A_{l+1}, \ldots, A_{v}\right\}$, where $0<\mu\left(C_{s}\right)<$ $+\infty$ for $s=1, \ldots, p$ and $\mu\left(D_{t}\right)=0$ for $t=1, \ldots, q$. (The union $\bigcup_{i=1}^{n} A_{i}$ has finite measure because it is a finite union of sets of finite measure.) For every $s=1, \ldots, h$, the set $B_{s}$ includes at least one piece $C_{j}$. Moreover, since $B_{1}, \ldots, B_{h}$ are pairwise disjoint, by re-arranging if necessary, we can also assume that $C_{s} \subseteq B_{s}$ for $s=1, \ldots, h$.

We now need the following result:

- "Given finitely many real numbers $y_{s}>0$, for every $\varepsilon>0$ there exists $N \in \mathbb{N}$ such that every fractional part $\left\{N \cdot y_{s}\right\}=N \cdot y_{s}-\left[N \cdot y_{s}\right]<\varepsilon$ ".

Recall that by Dirichlet's simultaneous approximation theorem (see eg Hardy and Wright $[9, \S 11.12])$, the above property holds if at least one of the $y_{s}$ is irrational. On the other hand, when all $y_{s} \in \mathbb{Q}$, if $N$ is any multiple of the greatest common denominator of the numbers $y_{s}$, then trivially all fractional parts $\left\{N \cdot y_{s}\right\}=0$.

Let

- $\alpha=\mu\left(\bigcup_{i=1}^{n} A_{i}\right)$;

- $c=\min \left\{\mu\left(C_{s}\right) \mid s=1, \ldots, p\right\}$.

By the above property we can pick a natural number $N$ such that:

(a) $N>\frac{\alpha(2 m+1)(k+1)}{c^{2}}$;

(b) $e_{s}=\left\{N \cdot \mu\left(C_{s}\right)\right\}<\frac{1}{p}$ for all $s=1, \ldots, p$.

Denote by

- $C=\bigsqcup_{s=1}^{p} C_{s}$ the "relevant part" of the partition ;

${ }^{3}$ Recall that the partition induced by a finite family $\left\{A_{1}, \ldots, A_{n}\right\}$ is the partition on $A_{1} \cup \ldots \cup A_{n}$ given by the nonempty intersections $\bigcap_{i=1}^{n} A_{i}^{\chi(i)}$ for $\chi:\{1, \ldots, n\} \rightarrow\{-1,1\}$, where $A_{j}^{1}=A_{j}$ and $A_{j}^{-1}=\left(\bigcup_{i=1}^{n} A_{i}\right) \backslash A_{j}$. 
- $D=\bigsqcup_{t=1}^{q} D_{t}$ the "negligible part" of the partition;

- $F=\left\{x_{1}, \ldots, x_{k}\right\}$.

Then, set

- $N_{s}=\left[N \cdot \mu\left(C_{s}\right)\right]$ for $s=1, \ldots, p$;

- $M_{s}=\left|B_{s} \cap D \cap F\right|$ for $s=1, \ldots, h$.

Notice that $N_{s}>k$ for all $s$. In fact, by the above conditions $(a)$ and $(b)$ :

$$
\begin{aligned}
N_{s} & =N \cdot \mu\left(C_{s}\right)-e_{s}>\frac{\alpha(2 m+1)(k+1)}{c^{2}} \cdot \mu\left(C_{s}\right)-e_{s}> \\
& >\frac{\alpha \cdot \mu\left(C_{s}\right)}{c^{2}} \cdot(k+1)-e_{s}>1 \cdot(k+1)-1=k .
\end{aligned}
$$

For $s=1, \ldots, h$, pick a finite subset $\lambda_{s} \subset C_{s}$ containing exactly $\left(N_{s}-M_{s}\right)$-many elements, and such that $C_{s} \cap F \subseteq \lambda_{s}$. Observe that this is possible because

$$
\left|C_{s} \cap F\right| \leq\left|B_{s} \cap C \cap F\right|=\left|B_{s} \cap F\right|-M_{s} \leq k-M_{s}<N_{s}-M_{s} .
$$

For $s=h+1, \ldots, p$, pick a finite subset $\lambda_{s} \subset C_{s}$ containing exactly $N_{s}$-many elements. Finally, define

$$
\lambda=F \cup \bigcup_{s=1}^{p} \lambda_{s} .
$$

We claim that $\lambda$ satisfies the desired properties (1), (2), (3). Since $F \subseteq \lambda$, condition (1) is trivially satisfied. For every $i=1, \ldots, n$ let:

$$
G(i)=\left\{s \leq h \mid C_{s} \subseteq A_{i}\right\} \quad \text { and } \quad G^{\prime}(i)=\left\{s>h \mid C_{s} \subseteq A_{i}\right\} .
$$

With the above definitions, we obtain:

$$
\begin{aligned}
\left|\lambda \cap A_{i}\right| & =\sum_{s \in G(i)}\left|\lambda_{s}\right|+\sum_{s \in G^{\prime}(i)}\left|\lambda_{s}\right|+\left|A_{i} \cap D \cap F\right| \\
& =\sum_{s \in G(i)}\left(N_{s}-M_{s}\right)+\sum_{s \in G^{\prime}(i)} N_{s}+\left|A_{i} \cap D \cap F\right| \\
& =\sum_{s \in G(i) \cup G^{\prime}(i)} N_{s}-\sum_{s \in G(i)} M_{s}+\left|A_{i} \cap D \cap F\right| \\
& =N \cdot\left(\sum_{s \in G(i) \cup G^{\prime}(i)} \mu\left(C_{s}\right)\right)-\varepsilon_{i}-\eta_{i}+\vartheta_{i} \\
& =N \cdot \mu\left(A_{i}\right)-\varepsilon_{i}-\eta_{i}+\vartheta_{i}
\end{aligned}
$$

where: 
- $\varepsilon_{i}=\sum_{s \in G(i) \cup G^{\prime}(i)} e_{s} \leq \sum_{s=1}^{p} e_{s}<1$ by condition $(b)$;

- $\eta_{i}=\sum_{s \in G(i)} M_{s} \leq \sum_{s=1}^{h}\left|B_{s} \cap D \cap F\right| \leq|F|=k$;

- $\vartheta_{i}=\left|A_{i} \cap D \cap F\right| \leq k$.

If $A_{i} \in \mathfrak{B}_{f}$, i.e. if $i \leq l$, recall that $A_{i}=\bigsqcup_{s \in S(i)} B_{s}$ for a suitable $S(i) \subseteq\{1, \ldots, h\}$. Since $C_{s} \subseteq B_{s}$ for all $s=1, \ldots, h$, it must be $G(i)=S(i)$. So, for $i \leq l$ we have

$$
\begin{aligned}
\eta_{i} & =\sum_{s \in S(i)} M_{s}=\sum_{s \in S(i)}\left|B_{s} \cap D \cap F\right|=\left|\left(\bigsqcup_{s \in S(i)} B_{s}\right) \cap D \cap F\right| \\
& =\left|A_{i} \cap D \cap F\right|=\vartheta_{i},
\end{aligned}
$$

and hence $\left|\lambda \cap A_{i}\right|=N \cdot \mu\left(A_{i}\right)-\varepsilon_{i}$. In consequence, for every $i, j \leq l$ such that $\mu\left(A_{i}\right)=\mu\left(A_{j}\right)$, one has that

$$
|| \lambda \cap A_{i}|-| \lambda \cap A_{j}||=\left|N \cdot \mu\left(A_{i}\right)-\varepsilon_{i}-N \cdot \mu\left(A_{j}\right)+\varepsilon_{j}\right|=\left|\varepsilon_{j}-\varepsilon_{i}\right| .
$$

Now notice that $\left|\varepsilon_{j}-\varepsilon_{i}\right| \leq \max \left\{\varepsilon_{i}, \varepsilon_{j}\right\}<1$, and so the natural numbers $\left|\lambda \cap A_{i}\right|=$ $\left|\lambda \cap A_{j}\right|$ necessarily coincide. This completes the proof of (2).

As for (3), notice that $\left|\lambda \cap A_{i}\right|=N \cdot \mu\left(A_{i}\right)+\zeta_{i}$ where $\zeta_{i}=\left(\vartheta_{i}-\eta_{i}\right)-\varepsilon_{i}$ is such that $-(k+1)<\zeta_{i} \leq k$. For every $i, j$ such that $\mu\left(A_{j}\right) \neq 0$, we have that

$$
\frac{N \cdot \mu\left(A_{i}\right)+\zeta_{i}}{N \cdot \mu\left(A_{j}\right)+\zeta_{j}}-\frac{\mu\left(A_{i}\right)}{\mu\left(A_{j}\right)}=\frac{\mu\left(A_{j}\right) \cdot \zeta_{i}-\mu\left(A_{i}\right) \cdot \zeta_{j}}{N \cdot \mu\left(A_{j}\right)^{2}+\mu\left(A_{j}\right) \cdot \zeta_{j}} .
$$

Now, the absolute value of the numerator

$$
\left|\mu\left(A_{j}\right) \cdot \zeta_{i}-\mu\left(A_{i}\right) \cdot \zeta_{j}\right|<\left(\mu\left(A_{i}\right)+\mu\left(A_{j}\right)\right) \cdot(k+1) \leq 2 \alpha(k+1) ;
$$

and the absolute value of the denominator

$$
\begin{aligned}
\left|N \cdot \mu\left(A_{j}\right)^{2}+\mu\left(A_{j}\right) \cdot \zeta_{j}\right|>N c^{2}-\alpha(k+1) & >\alpha(2 m+1)(k+1)-\alpha(k+1)=2 m \alpha(k+1) .
\end{aligned}
$$

So, we reach the thesis:

$$
\left|\frac{\left|\lambda \cap A_{i}\right|}{\left|\lambda \cap A_{j}\right|}-\frac{\mu\left(A_{i}\right)}{\mu\left(A_{j}\right)}\right|<\frac{2 \alpha(k+1)}{2 m \alpha(k+1)}=\frac{1}{m} .
$$

In order to get a finite subset $\lambda^{\prime}$ satisfying also property (4), for $t=n+1, \ldots, v$ pick a finite subset $\eta_{t} \subset A_{t} \backslash \bigcup_{i=1}^{n} A_{i}$ with $\left|\eta_{t}\right|>m \cdot|\lambda|$, and set $\lambda^{\prime}=\lambda \cup \bigcup_{t=n+1}^{v} \eta_{t}$. It is clear that such a $\lambda^{\prime}$ still satisfies properties (1), (2), (3), and it is readily checked that it also satisfies property (4). 
Proof of Theorem 2.2 Let $\Lambda=\operatorname{Fin}(\Omega)$ be the family of all finite subsets of $\Omega$, and define the following subsets of $\Lambda$ :

- For all $x \in \Omega$, let

$$
\widehat{x}=\{\lambda \in \Lambda: x \in \lambda\} .
$$

- For all $A, A^{\prime} \in \mathfrak{A}_{f}$ with $\mu\left(A^{\prime}\right)>0$ and for all $n \in \mathbb{N}$, let

$$
\Gamma\left(A, A^{\prime}, n\right)=\left\{\lambda \in \Lambda: \lambda \cap A^{\prime} \neq \emptyset \text { and }\left|\frac{|\lambda \cap A|}{\left|\lambda \cap A^{\prime}\right|}-\frac{\mu(A)}{\mu\left(A^{\prime}\right)}\right|<\frac{1}{n}\right\} .
$$

- For all nonempty $B, B^{\prime} \in \mathfrak{B}_{f}$, let

$$
\Theta\left(B, B^{\prime}\right)=\left\{\lambda \in \Lambda:|B \cap \lambda|=\left|B^{\prime} \cap \lambda\right|\right\} .
$$

- For all $C \in \mathfrak{A} \backslash \mathfrak{A}_{f}$, for all $C^{\prime} \in \mathfrak{A}_{f}$ with $\mu\left(C^{\prime}\right)>0$, and for all $n \in \mathbb{N}$, let

$$
\Xi\left(C, C^{\prime}, n\right)=\left\{\lambda \in \Lambda: \lambda \cap C^{\prime} \neq \emptyset \text { and } \frac{|\lambda \cap C|}{\left|\lambda \cap C^{\prime}\right|}>n\right\} .
$$

Then consider the following family:

$$
\begin{aligned}
\mathcal{G}= & \{\widehat{x} \mid x \in \Omega\} \bigcup\left\{\Gamma\left(A, A^{\prime}, n\right) \mid A, A^{\prime} \in \mathfrak{A}_{f}, \mu\left(A^{\prime}\right)>0, n \in \mathbb{N}\right\} \\
& \bigcup\left\{\Theta\left(B, B^{\prime}\right) \mid B, B^{\prime} \in \mathfrak{B}_{f}, \mu(B)=\mu\left(B^{\prime}\right)>0\right\} \\
& \bigcup\left\{\Xi\left(C, C^{\prime}, n\right) \mid C \in \mathfrak{A}^{\prime} \backslash \mathfrak{A}_{f}, C^{\prime} \in \mathfrak{A}_{f}, \mu\left(C^{\prime}\right)>0, n \in \mathbb{N}\right\} .
\end{aligned}
$$

As a consequence of the Lemma, the family $\mathcal{G}$ has the finite intersection property. Indeed, let finitely many elements of $\mathcal{G}$ be given, say

$$
\begin{gathered}
\widehat{x}_{1}, \ldots, \widehat{x}_{k} ; \Gamma\left(A_{1}, A_{1}^{\prime}, n_{1}\right), \ldots, \Gamma\left(A_{u}, A_{u}^{\prime}, n_{u}\right) ; \\
\Theta\left(B_{1}, B_{1}^{\prime}\right), \ldots, \Theta\left(B_{w}, B_{w}^{\prime}\right) ; \Xi\left(C_{1}, C_{1}^{\prime}, m_{1}\right), \ldots, \Xi\left(C_{s}, C_{s}^{\prime}, m_{s}\right) .
\end{gathered}
$$

Without loss of generality, we can assume that the set $\left\{x_{1}, \ldots, x_{k}\right\}$ includes at least one point from each of the above sets $A_{i}^{\prime}$ and $C_{j}^{\prime}$. Pick $m=\max \left\{n_{1}, \ldots, n_{u}, m_{1}, \ldots, m_{s}\right\}$ and apply Lemma 3.1 to get the existence of a finite set $\lambda \in \Lambda$ such that:

(1) $x_{1}, \ldots, x_{k} \in \lambda$;

(2) For all $i=1, \ldots, w$, if $\mu\left(B_{i}\right)=\mu\left(B_{i}^{\prime}\right)$ then $\left|\lambda \cap B_{i}\right|=\left|\lambda \cap B_{i}^{\prime}\right|$;

(3) For all $i, j=1, \ldots, u$, then

$$
\left|\frac{\left|\lambda \cap A_{j}\right|}{\left|\lambda \cap A_{i}^{\prime}\right|}-\frac{\mu\left(A_{j}\right)}{\mu\left(A_{i}^{\prime}\right)}\right|<\frac{1}{m} ;
$$


(4) for all $i, j=1, \ldots, s$ :

$$
\frac{\left|\lambda \cap C_{i}\right|}{\left|\lambda \cap C_{j}^{\prime}\right|}>m
$$

Then it is readily verified that such a $\lambda$ belongs to all considered sets of $\mathcal{G}$. Since $|\mathcal{G}| \leq 2^{|\Omega|}$, we can apply the enlarging property and pick a set

$$
F \in \bigcap_{G \in \mathcal{G}}{ }^{*} G
$$

Now notice that:

(a) For every $x \in \Omega, F \in{ }^{*} \widehat{x}$ means that ${ }^{*} x \in F$;

(b) For every $A, A^{\prime} \in \mathfrak{A}_{f}$ with $\mu\left(A^{\prime}\right)>0, F \in{ }^{*} \Gamma\left(A, A^{\prime}, n\right)$ for every $n \in \mathbb{N}$ means that

$$
\frac{\|F \cap * A\|}{\left\|F \cap{ }^{*} A^{\prime}\right\|} \approx \frac{\mu(A)}{\mu\left(A^{\prime}\right)}
$$

(c) For every $B, B^{\prime} \in \mathfrak{B}_{f}$ with $\mu(B)=\mu\left(B^{\prime}\right)>0, F \in{ }^{*} \Theta\left(B, B^{\prime}\right)$ means that $\left\|F \cap{ }^{*} B\right\|=\left\|F \cap{ }^{*} B^{\prime}\right\|$;

(d) For every $C, C^{\prime} \in \mathfrak{A}$ with $\mu(C)=+\infty$ and $0<\mu\left(C^{\prime}\right)<+\infty, F \in{ }^{*} \Xi\left(C, C^{\prime}, n\right)$ for every $n \in \mathbb{N}$ means that

$$
\frac{\left\|F \cap{ }^{*} C\right\|}{\left\|F \cap{ }^{*} C^{\prime}\right\|} \text { is infinite. }
$$

Properties (1) and (2) of the thesis are directly given by $(a)$ and $(c)$, respectively. As for (3), let $\beta=\left\|F \cap{ }^{*} Z\right\| / \mu(Z)$ where $Z \in \mathfrak{A}$ is such that $0<\mu(Z)<+\infty$. If $\mu(A)<+\infty$, by property $(b)$ where $A^{\prime}=Z$, we get

$$
\frac{\left\|F \cap{ }^{*} A\right\|}{\beta}=\frac{\left\|F \cap{ }^{*} A\right\|}{\left\|F \cap{ }^{*} Z\right\|} \cdot \mu(Z) \approx \frac{\mu(A)}{\mu(Z)} \cdot \mu(Z)=\mu(A) ;
$$

and if $\mu(A)=+\infty$, by property $(d)$ where $C=A$ and $C^{\prime}=Z$, we have that the following number is infinite:

$$
\frac{\left\|F \cap{ }^{*} A\right\|}{\beta}=\frac{\left\|F \cap \cap^{*} A\right\|}{\left\|F \cap \cap^{*} Z\right\|} \cdot \mu(Z) .
$$

\section{References}

[1] V. Benci, I numeri e gli insiemi etichettati, Conferenza del seminario di matematica dell'Università di Bari, vol. 261 (1995), Laterza, 1-29. 
[2] V. Benci, E. Bottazzi, M. Di Nasso, Some applications of numerosities in measure theory, in preparation.

[3] V. Benci, M. Di Nasso , Numerosities of labelled sets: a new way of counting, Advances in Mathematics, vol. 173 (2003), 50-67, doi: http : //dx . doi .org/10.1016/ S0001-8708(02)00012-9.

[4] V. Benci, M. Di Nasso, M. Forti, An Aristotelian notion of size, Annals of Pure and Applied Logic, vol. 143 (2006), 43-53, doi: http://dx.doi.org/10.1016/j . apal.2006.01.008.

[5] A. Bernstein, F. Wattenberg, Nonstandard measure theory, in Applications of Model Theory to Algebra, Analysis and Probability (W.A.J. Luxemburg, ed.), Holt, Rinehart and Winston (1969), 171-185.

[6] M. Davis, Applied Nonstandard Analysis, John Wiley \& Sons (1977).

[7] M. Di Nasso, M. Forti, Numerosities of point sets over the real line, Transactions of the American Mathematical Society, vol. 362 (2010), 5355-71, doi: http://dx . doi . org/10.1090/S0002-9947-2010-04919-0.

[8] R. Goldblatt, Lectures on the Hyperreals - An Introduction to Nonstandard Analysis, Graduate Texts in Mathematics, vol. 188 (1998), Springer, doi: http: //dx .doi .org/ 10.1007/978-1-4612-0615-6.

[9] G.H. Hardy, E.M. Wright, An Introduction to the Theory of Numbers (6th edition, R. Heath-Brown, J. Silverman, A. Wiles, eds.), Oxford University Press (2008).

[10] C.W. Henson, On the nonstandard representation of measures, Transactions of the American Mathematical Society, vol. 172 (1972), 437-446, doi: http://dx.doi. org/10.1090/S0002-9947-1972-0315082-2.

[11] J. Yeh, Real Analysis, Theory of Measure and Integration, World Scientific Publishing Co. Pte. Ltd (2006), doi: http://dx.doi .org/10.1142/6023.

[12] P.A. Loeb, Conversion from nonstandard to standard measure spaces and applications in probability theory, Transactions of the American Mathematical Society, vol. 211 (1975), 113-122, doi: http://dx.doi.org/10.1090/ S0002-9947-1975-0390154-8.

[13] A. Robinson, On generalized limits and linear functionals, Pacific Journal of Mathematics, vol. 14 (1975), 269-283, doi: http://dx.doi.org/10.2140/pjm. 1964 . 14. 269.

[14] D.A. Ross, Loeb measure and probability, in Nonstandard Analysis - Theory and Applications (L.O. Arkeryd, N.J. Cutland, C.W. Henson, eds.), NATO ASI Series C, vol. 493 (1997), Kluwer A.P., pp. 91-120, doi: http://dx.doi.org/10.1007/ 978-94-011-5544-1_4.

[15] D.A. Ross, Nonstandard measure constructions - Solutions and problems, in Nonstandard Methods and Applications in Mathematics (N.J. Cutland, M. Di Nasso, D.A. Ross, eds.), Lecture Notes in Logic, vol. 25 (2006), Association of Symbolic Logic, pp. 127-146. 
Università di Pisa, Italy and Department of Mathematics, College of Science, King Saud University, Riyadh, Saudi Arabia.

Dipartimento di Matematica, Università di Trento, Italy.

Dipartimento di Matematica, Università di Pisa, Italy.

benci@dma.unipi.it, emanuele.bottazzi@unitn.it, dinasso@dm.unipi.it

http://www.dma.unipi.it/Members/benci,

http://www.science.unitn.it/ bottazzi/,

http://www.dm.unipi.it/ dinasso/

Received: 17 October $2013 \quad$ Revised: 13 August 2014

Journal of Logic \& Analysis 6:3 (2014) 